\title{
On-Demand Preparation of Microdot Patterns by Laser-Induced Dot Transfer
}

\author{
Aiko NARAZAKI, Ryozo KUROSAKI, Tadatake SATO, Yoshizo KAWAGUCHI, \\ Wataru WATANABE and Hiroyuki NIINO

\begin{abstract}
Electronics and Photonics Research Institute, National Institute of Advanced Industrial Science and Technology (AIST), Central 5, 1-1-1 Higashi, Tsukuba, Ibaraki 305-8565, Japan
\end{abstract} \\ E-mail:narazaki-aiko@aist.go.jp
}

\begin{abstract}
Flexible and rapid preparation of $\mathrm{FeSi}_{2}$ microdots and indium tin oxide (ITO) microstructure patterns was performed using laser-induced forward transfer with a nanosecond high-frequency diode-pumped solid-state laser/galvanometer-based point-scanning system. Different types of source materials, i.e., $\mathrm{FeSi}_{2}$ and ITO, were used to explore the materials dependence of transfer. $\mathrm{FeSi}_{2}$ spheres with a lateral diameter of $5.5 \mu \mathrm{m}$ were successfully arrayed on a micrometer scale. Sub-spot transfer was achieved, and we refer to this as laser-induced dot transfer (LIDT). In the case of ITO, although the transferred shape is flower-like rather than spherical, site-controlled transfer was achieved. The LIDT technique is useful for integrating functional micro-patterns with sub-spot resolution under room-temperature atmospheric conditions.

DOI:10.2961/jlmn.2012.01.0015
\end{abstract}

Keywords: laser-induced dot transfer, on-demand preparation, microdot pattern, $\mathrm{FeSi}_{2}$, ITO

\section{Introduction}

Laser-induced forward transfer (LIFT) has been studied extensively as a method for additive micro-patterning because it has the advantage of being a simple atmospheric low-temperature process [1-3]. Recent LIFT works have focused on two main features: deposition that is less damaging to target materials, and sub-spot transfer resulting in typical droplet volumes of from a tenth to several femtoliters [4-8]. The former feature is favorable for patterning biomaterials [9-12] and integrating functional materials on soft substrates such as organic semiconductors and metal electrodes for organic thin-film transistors (OTFTs) [13, 14]. In the latter feature, i.e., sub-spot transfer, one-to-one dot deposition with a laser-illuminated area is used; we refer to this as laser-induced dot transfer (LIDT) [6] to distinguish it from conventional LIFT. The LIDT process provides a new way of micro-patterning a variety of materials, with higher resolutions than those obtained using commercially available inkjet techniques.

We had already developed an LIDT technique using a nanosecond excimer laser/mask-projection system [6, 7]. The main advantage of this system is size-controlled deposition of submicron- and micro-dots in numbers of more than ten thousand by single-pulse irradiation. $\mathrm{FeSi}_{2}$ submicron- and micro-dots were prepared in association with room-temperature (RT) precipitation of $\beta-\mathrm{FeSi}_{2}$ semiconducting crystallites. Near-infrared (NIR) photoluminescence was successfully detected from these dots [7]. The semiconductor $\beta-\mathrm{FeSi}_{2}$ is one of the potential candidates for Si-based light emitters and detectors at 1.5-1.6 $\mu \mathrm{m}$ in the NIR suitable for optical networking, solar cells, and thermoelectric devices $[15,16]$. $\beta$-FeSi $i_{2}$ has other excellent features such as the rich abundance of its constituents in natural resources and non-toxicity, making it an "ecofriendly" semiconductor. $\beta-\mathrm{FeSi}_{2}$ film preparation generally requires high-temperature processing at $450-800{ }^{\circ} \mathrm{C}$ to achieve crystallization in the $\beta$ phase. However, experience suggests that melting via the LIDT process may lead to RT precipitation of the $\beta$ crystalline phase; this is an additional advantage of the LIDT process.

In this work, we report flexible and rapid direct-writing of microdot patterns via LIDT with our high-frequency nanosecond diode-pumped solid-state (DPSS) ultraviolet laser/galvanometer-based point-scanning system. Different types of source materials, i.e., $\mathrm{FeSi}_{2}$ and $\mathrm{In}_{2} \mathrm{O}_{3}-\mathrm{SnO}_{2}$ (indium tin oxide, ITO), were used to explore the materials dependence of dot transfer. Furthermore, the LIDT mechanism was investigated by subjecting the $\mathrm{FeSi}_{2}$ source film to the LIDT process with a $\mathrm{KrF}$ excimer laser/maskprojection system.

\section{Experimental}

The third harmonic of an Nd:YVO ${ }_{4}$ DPSS laser $(\lambda=$ $355 \mathrm{~nm}$, full-width at half-maximum (FWHM) $30 \mathrm{~ns}, M^{2}<$ 1.3) was used as a light source. The laser was operated at a repetition rate of $5 \mathrm{kHz}$. Laser pulses of spot size approximately $14 \mu \mathrm{m}$ were scanned with a galvanometer-based point-scanning module (GSI Lumonics; HPM10M2) for on-demand microdot preparation using the LIDT process, as shown in Fig. 1. In the LIDT event, a single pulse was focused at the interface between a transparent support (silica glass in this work) and the source film ( $\mathrm{FeSi}_{2}$ or ITO). Amorphous $\mathrm{FeSi}_{2}$ and ITO source films were prepared on silica glass plates by RF magnetron sputtering. The film thickness was 500-600 nm. The incident laser pulse power was set at 5 and $2 \mu \mathrm{J} \cdot$ pulse $^{-1}$ in the cases of $\mathrm{FeSi}_{2}$ and ITO, 


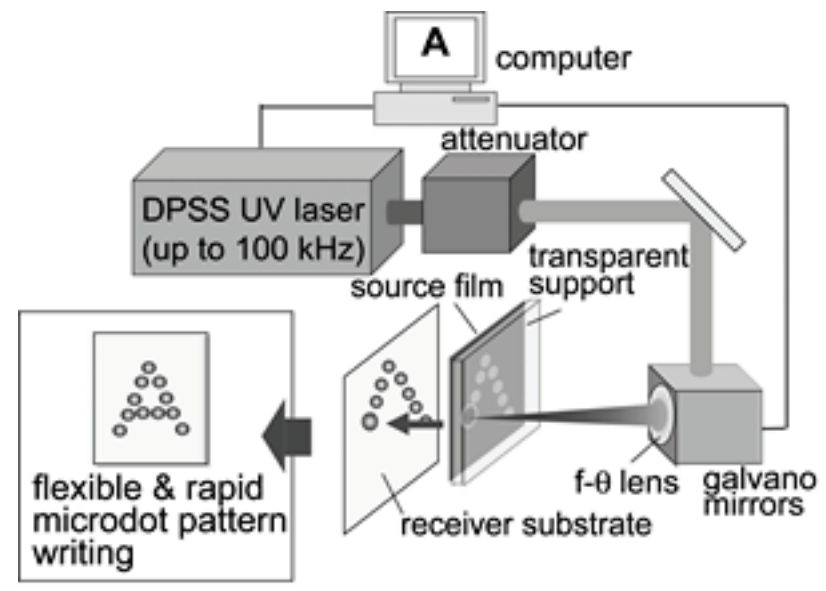

Fig. 1 Setup for on-demand microdot preparation by laserinduced transfer. A single pulse leads to deposition of a single microdot on a receiver substrate. For flexible and rapid dot pattering, laser pulses from a high-frequency DPSS laser were scanned on a source film using a galvanometer-based point-scanning system.

respectively. As a receiver substrate, ozone-cleaned silica glass was placed opposite the source film at a distance of $\sim 0$ (nearly-contact) or $30 \mu \mathrm{m}$ (non-contact, with a spacer). After single-pulse irradiation at an optimum fluence, a single microdot is deposited onto the receiver substrate.

The surface morphologies of the thus-obtained samples were observed using a scanning electron microscope (SEM; Keyence, VE-7800) and a confocal scanning laser microscope (Keyence, VK-8500). The cross-sectional profile was also obtained by laser microscopy.

In order to investigate the LIDT mechanism, SEM observations were conducted for the $\mathrm{FeSi}_{2}$ source film after the LIDT with a $\mathrm{KrF}$ excimer laser/mask-projection system $[6,7]$.

\section{Results}

Figure 2 shows a $\mathrm{FeSi}_{2}$ microdots array prepared on a silica glass substrate using the galvanometer-based pointscanning module in Fig. 1. The $\mathrm{FeSi}_{2}$ alloy source film was irradiated using a single laser-pulse with a spot diameter of

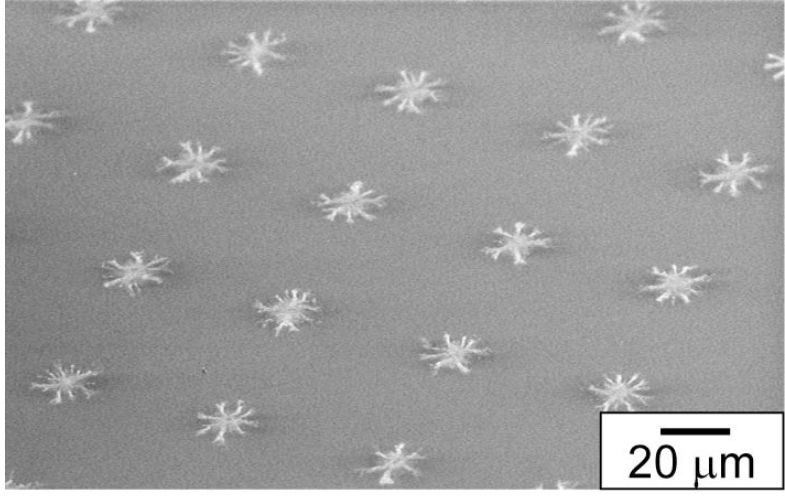

Fig. 3 SEM image of ITO microstructures array on a silica glass substrate.

$14 \mu \mathrm{m}$, leading to deposition of a spherical dot of diameter approximately $5.5 \mu \mathrm{m}$ in the lateral direction. The gap between the source film and the receiver was $30 \mu \mathrm{m}$. Since the dot contacts the substrate at its bottom face, it is not a perfect sphere. Thus, the transferred volume is less than the volume of $87 \mu^{3}$ of the perfect sphere. The laserirradiated film volume is estimated to be from 77 to $92 \mu \mathrm{m}^{3}$ for films of thickness $500-600 \mathrm{~nm}$. From these results, it is obvious that the dot volume is comparable to the laserirradiated film volume. The morphology changes in the transferred material indicate melting of the source film in the laser-irradiated volume.

Figure 3 shows an ITO microstructures array on a silica glass substrate obtained using the system in Fig. 1. Unlike the $\mathrm{FeSi}_{2}$ microdots in Fig. 2, the deposited microstructure has a flower-like shape. The laser fluence for transfer was smaller than that for $\mathrm{FeSi}_{2}$ and the gap between the source film and the receiver substrate was set at nearly-contact, without a spacer. An increase in the laser fluence easily caused film fracture and degraded the transfer properties. Even at the optimum fluence, in the case of non-contact mode with a spacer, no transfer was observed. Although the shape of the transferred microstructure changed, sitecontrolled deposition was successfully achieved for both $\mathrm{FeSi}_{2}$ and ITO.

Figure 4 shows the confocal scanning laser microscopic image and profile for the $\mathrm{FeSi}_{2}$ source film after the LIDT

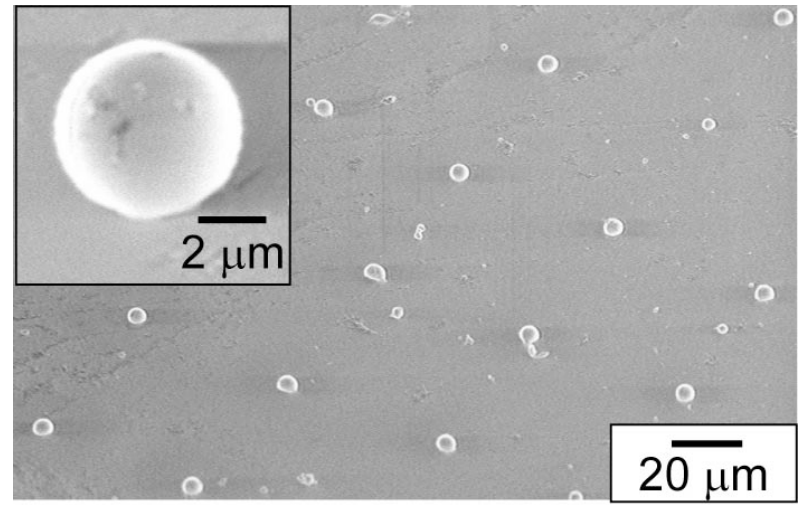

Fig. 2 SEM images of $\mathrm{FeSi}_{2}$ microdots array on a silica glass substrate. The transfer system shown in Fig. 1 was used for on-demand dot preparation.

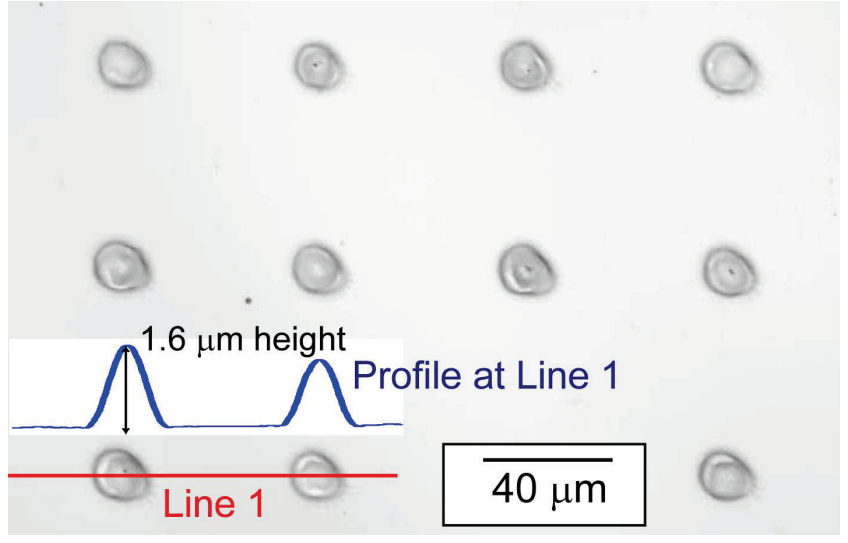

Fig. 4 Confocal scanning laser microscopic image and profile of a $\mathrm{FeSi}_{2}$ source film after one-to-one dot deposition. The profile at Line 1 shows that the laser spots tend to become convex with heights over $1 \mu \mathrm{m}$. 


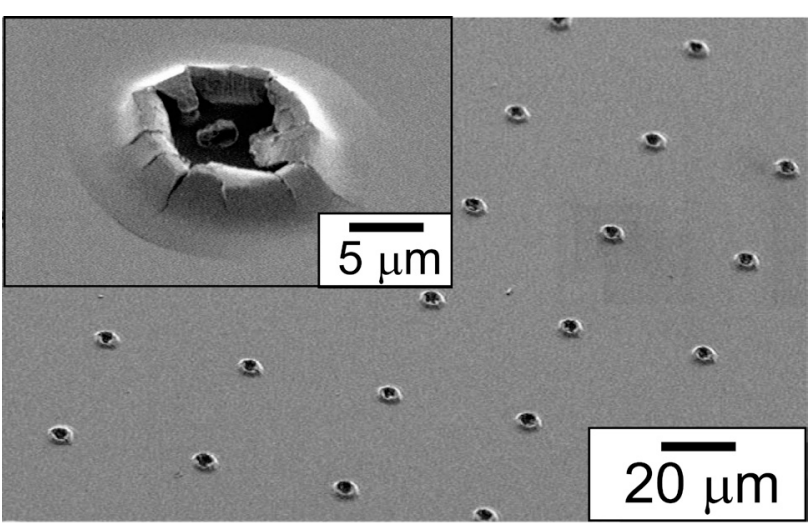

Fig. 5 SEM images of ITO source films after laser irradiation. In the upper magnified picture, the rim contour clearly exhibits many cracks, unlike the $\mathrm{FeSi}_{2}$ source film shown in Fig. 4.

process. The resultant microdots array is shown in Fig.2. Most of the spots became convex with a height of approximately $1.6 \mu \mathrm{m}$. This tendency is similar to that for the morphology of a film irradiated through an excimer laser/ mask-projection system [6]. When the laser fluence increased, the spot morphology changed from convex to through-hole. A further fluence increase resulted in splashing, which prevented site- and size-controlled dot depositions. However, fracture of the $\mathrm{FeSi}_{2}$ source film did not occur.

SEM images of the ITO source film after the laserinduced transfer process are shown in Fig. 5. The laser irradiation spots are through-holes and have rims. The corresponding transfer structures are shown in Fig. 3. Unlike the $\mathrm{FeSi}_{2}$ source film in Fig. 4, the rims show several cracks.

For the purpose of exploring a possible mechanism for the LIDT process, an amorphous $\mathrm{FeSi}_{2}$ source film was subjected to the LIDT process with a $\mathrm{KrF}$ excimer laser single-pulse. The gap between the source film and a silicon wafer receiver substrate was set at nearly contact, without a

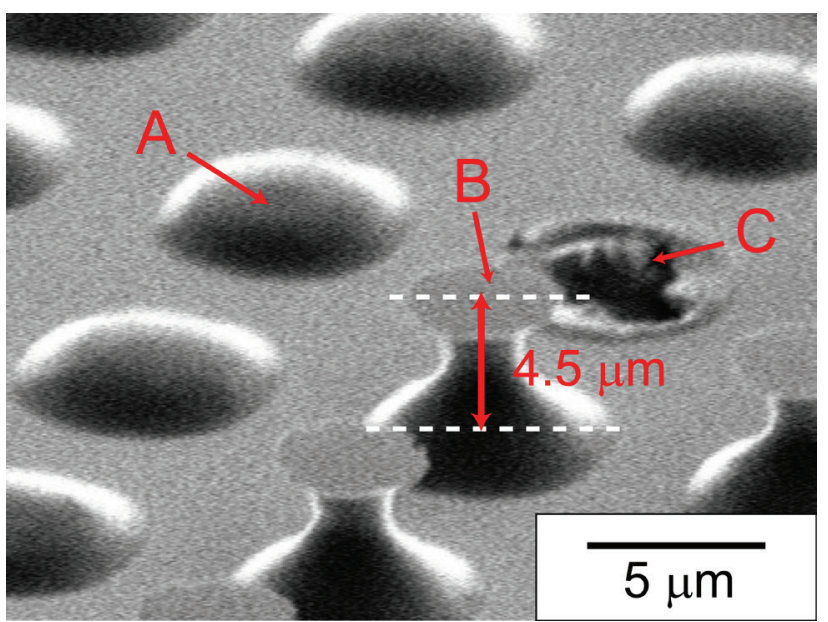

Fig. $6 \mathrm{SEM}$ image of a $\mathrm{FeSi}_{2}$ source film after single-pulse irradiation through a mask-projection system. A $\mathrm{KrF}$ excimer laser was used as a light source. Although most of the laser spots are convex, like "A," a few spots have a unique structure like "B" or a through-hole structure "C." spacer. Figure 6 shows the surface morphology of the source film Three kinds of spot morphology were observed: a convex part, denoted by "A," a unique structure, denoted by "B," and a through-hole, denoted by "C." This variety possibly stems from small fluctuations in the fluence at the edge of the imaging area. In fact, most of the spots in the center of the imaging area exhibit convex " $\mathrm{A}$ " structures. Structure "B" has a height of about $4.5 \mu \mathrm{m}$. This height might correspond to the native gap between the film and receiver when they were placed in our system without a spacer. With increasing laser fluence, structure " $\mathrm{C}$ " becomes the predominant structure.

\section{Discussion}

Site-controlled deposition of microstructures was achieved, irrespective of the materials used, by laserinduced transfer with a high-frequency nanosecond DPSS laser/galvanometer-based point-scanning system, as shown in Figs. 2 and 3. In the case of $\mathrm{FeSi}_{2}$, in particular, the diameter of the transferred dot was much smaller than the laser spot, indicating that LIDT was successfully achieved.

However, the lateral size of the ITO microstructure was comparable to that of the laser spot and it was not spherical, unlike the shape generally observed for LIDT of metals and $\mathrm{FeSi}_{2}$ in this work. These facts suggest that the present ITO transfer is not so much LIDT as general LIFT. The difference in shape can be explained as follows. The ITO film is more brittle than normal metal films. The existence of obvious cracks at the rim in the microscopic images of the laser-irradiated area shown in Fig. 5 confirms the brittleness of the ITO [17]. This brittleness easily leads to film fracture with increasing laser fluence, making the LIDT process difficult at higher fluences. High-quality dot transfer with an ITO film is therefore limited in the nearlycontact mode operated in a relatively low fluence range. Taking into account the reflectance at the interface between the silica glass support and the source film, the optimum pulse power incident upon the $\mathrm{FeSi}_{2}$ film was calculated to be $3.4 \mu \mathrm{J} \cdot$ pulse $^{-1}$, approximately twice that for ITO. The incidence of the lower laser power possibly prevents melting in the entire laser-irradiated area in the case of ITO. The shape of the transferred microstructure is not spherical, like those produced from a melt, but has a unique structure, as shown in Fig. 3. One possible way to achieve the subspot transfer of oxide materials is laser irradiation with heating of the source film; this might decrease the thermal shock stress and prevent film fracture. Further investigations of size and shape control during laser-induced transfer of oxide materials are in progress, and the results will be reported in our future work.

Finally, taking the unique structure B in Fig. 6 into consideration, the possible LIDT mechanism is now discussed. Willis and Grosu simulated the melting behavior of aluminum under LIFT conditions [18]. They showed that a shallow melt zone was formed at a film/support interface, and then the melt zone continued to grow until it reached the free film surface. The free surface deformation resulting from volumetric expansion during the solid-liquid phase change was also simulated. Film deformation was clearly observed in the case of $\mathrm{FeSi}_{2}$, as shown in Fig. 4. Furthermore, in our previous work, a $\mathrm{FeSi}_{2}$ source film irradiated 
with a single $\mathrm{KrF}$ excimer laser pulse exhibited convex formations, as shown in Fig. 4. The source film was peeled from a transparent support for laser microscopic observations. These observations confirmed that the convex part was hollow and that its thickness was approximately 100 $\mathrm{nm}$, whereas the original film thickness was $500 \mathrm{~nm}$. It is therefore plausible that laser absorption at the film/support interface causes transient heating, melting of the film throughout its entire thickness, and film deformation, resulting in formation of a columnar-shaped jet with a diameter smaller than that of the laser spot. This jet touches the receiver substrate and leaves a microdot on the substrate, or detaches a droplet, which finally lands on the substrate, depending on the film/receiver distance. After material transfer, the remaining jet returns to the film surface, where solidification has already started, keeping the convex deformation. In the present work, we report the first experimental proof of such jet formation from a rigid solid film, i.e., structure "B" in Fig. 6. Structure "B" has a height corresponding to the native gap between the film and receiver substrate. It has a flat disk-like top. These features possibly indicate that when the laser-induced jet touched the silicon wafer surface, it was rapidly cooled as a result of the high thermal conductivity of silicon, and then solidified before returning to the source film surface. In fact, this kind of unique structure was not observed when silica glass was used as the receiver substrate. Jet formation is vital for high-quality dot preparation via LIDT.

\section{Conclusions}

On-demand preparation of microdots was performed using LIFT with a high-frequency nanosecond DPSS laser/ galvanometer-based point-scanning system for different kinds of materials, namely $\mathrm{FeSi}_{2}$ and ITO. $\mathrm{FeSi}_{2}$ spheres with a lateral diameter of $5.5 \mu \mathrm{m}$ were arrayed on a micrometer scale. Sub-spot transfer, which we call laserinduced dot transfer (LIDT), was therefore successfully achieved. In the case of ITO, although the transferred shape was not spherical but flower-like, site-controlled transfer was also achieved. Based on observation of the $\mathrm{FeSi}_{2}$ source film, jet formation was suggested as a possible LIDT mechanism. The LIDT technique is useful for integrating functional micro-patterns with sub-spot resolution under atmospheric RT conditions.

\section{References}

[1] J. Bohandy, B. F. Kim and F. J. Adrian: J. Appl. Phys., 60, (1986) 1538.

[2] I. Zergioti, S. Mailis, N. A. Vainos, P. Papakonstantinou, C. Kalpouzos, C. P. Grigoropoulos and C. Fotakis: Appl. Phys. A, 66, (1998) 579.

[3] A. Piqué, D. B. Chrisey, R. C. Y. Auyeung, J. FitzGerald, H. D. Wu, R. A. McGill, S. Lakeou, P. K. Wu, V. Nguyen and M. Duignan: Appl. Phys. A, 69, (1999) S279.

[4] D. A. Willis and V. Grosu, Appl. Phys. Lett., 86, (2005) 244103.

[5] D. P. Banks, C. Grivas, J. D. Mills, R. W. Eason and I. Zergioti: Appl. Phys. Lett., 89, (2006) 193107.

[6] A. Narazaki, T. Sato, R. Kurosaki, Y. Kawaguchi and H. Niino: Appl. Phys. Express, 1, (2008) 57001.

[7] A. Narazaki, T. Sato, R. Kurosaki, Y. Kawaguchi and H. Niino: Appl. Surf. Sci., 255, (2009) 9703.

[8] A. I. Kuznetsov, R. Kiyan and B. N. Chichkov: Opt. Express, 18, (2010) 21198.

[9] A. Karaiskou, I. Zergioti, C. Fotakis, M. Kapsetaki and D. Kafetzopoulos: Appl. Surf. Sci., 208-209, (2003) 245.

[10] P. Serra, M. Colina, J. M. Fernandez-Pradas, L. Sevilla and J. L. Morenza: Appl. Phys. Lett., 85, (2004) 1639.

[11] Y. Tsuboi, Y. Furuhata and N. Kitamura: Appl. Surf. Sci., 253, (2007) 8422.

[12] M. Duocastella, M. Colina, J. M. Fernandez-Pradas, P. Serra and J. L. Morenza: Appl. Surf. Sci., 253, (2007) 7855.

[13] L. Rapp, A. K. Diallo, A. P. Alloncle, C. VidelotAckermann, F. Fages and P. Delaporte: Appl. Phys. Lett., 95, (2009) 171109.

[14] H. Kim, R. C. Y. Auyeung, S. H. Lee, A. L. Huston and A. Piqué: Appl. Phys. A, 96, (2009) 441.

[15] D. Leong, M. Harry, K. J. Reeson and K. P. Homewood: Nature, 387, (1997) 686.

[16] T. Ootsuka, Y. Fudamoto, M. Osamura, T. Suemasu, Y. Makita, Y. Fukuzawa and Y. Nakayama: Appl. Phys. Lett., 91, (2007) 142114.

[17] J. Siegel, K. Ettrich, E. Welsch and E. Matthias: Appl. Phys. A, 64, (1997) 213.

[18]D. A. Willis and V. Grosu: Appl. Surf. Sci., 253, (2007) 475.

(Received: June 27, 2011, Accepted: January 7, 2012) 